\title{
Characteristic Parameters Extraction and Pattern Recognition of Partial Discharges based on Envelope of Ultra-high Frequency Signal
}

\author{
GAO Zhaoli", a , SUN Yingtao ${ }^{1, b}$, LUO Lingen ${ }^{2, c^{*}}$, SHENG Gehao ${ }^{2, d}$, and JIANG \\ Xiuchen $^{2, \mathrm{e}}$ \\ 1 Jinan Power Supply Company, Shandong Electric Power Corporation, Jinan, 250012, China \\ ${ }^{2}$ Department of Electrical Engineering, Shanghai Jiao Tong University, Shanghai City, 200240, \\ China \\ agzhl6360@163.com, b30706261@qq.com, 'Ilg523@sjtu.edu.cn, dshenghe@sjtu.edu.cn, \\ excjiang@sjtu.edu.cn
}

Keywords: gas insulated switchgear; partial discharge; ultra-high frequency; characteristic parameters; pattern recognition

Abstract. With the help of the Hilbert transform, the low-frequency signals modulated by high-frequency signals can be demodulated. This article proposed a method to extract the time-domain features of ultra-high frequency (UHF) signals regarding the partial discharges (PD) in a gas insulated switchgear (GIS). Utilizing the Hilbert transform, the envelope of a PD signal can be solved, and further the corresponding characteristic parameters of some key time domains can be acquired. An experimental platform for the detection of artificial insulation faults in a GIS was established. Thus, the experimental data of 3 types of typical PD signals were gained, and then used for the analysis and verification of the presented algorithm. The result shows that different types of PDs were accurately recognized. It can be concluded that the method presented is practical and effective.

\section{Introduction}

Gas insulated switchgear (GIS) exhibits a series of advantages such as small land occupation and high reliability, which has expanded its application in power systems [1]. However, the GIS's faults will bring about fairly serious consequences.

To monitor and identify the partial discharge (PD) in a GIS is an important means of finding the incipient insulation faults and preventing the accidents. Among various methods for monitoring the PD signals of GIS, the ultra-high frequency (UHF) method has high sensitivity and strong anti-interference performance; moreover, it can identify the fault type and precisely locate the PDs. The UHF method has become a hotspot in domestic and international studies during the past two decades $[2,3]$.

The insulation defects in a GIS have different types, such as the floating electrodes, free metallic particles and the contamination on the surface of insulators. Since different defects have various discharge characteristics and impose different degrees of damages on the GIS, an accurate assessment of the GIS's insulation status is of great importance.

Currently, the common method of identifying the PD types is based on the phase distribution spectra [4], without any consideration of the wave form information of PD pulses. Additionally, the phase synchronization information of power frequency is required.

In this article, we focused on another PD recognition method based on the waveform of PD pulses which requires no phase synchronization information regarding the power frequency. The main idea of this method is that the UHF signals of PDs can be regarded as the low frequency pulse signals modulated by the high frequency signals. The low frequency signals were demodulated using the Hilbert transform, and thus, the envelope of the PD signal was solved. Then the recognition of UHF PD signals can be achieved by extracting their corresponding envelope characteristics and combining a pattern recognition tool. 


\section{Envelope characteristic extraction for UHF signals}

The extraction of the envelope characteristics of UHF signals mainly includes the following three steps:

1) Filtering and denoising;

2) Acquisition of signal envelope;

3) Extraction of envelope characteristic parameters

\section{Filtering and denoising}

A spectral analysis was performed on three typical PD signals (namely, surface discharge, floating potential and metal particles), as shown in Fig. 1.

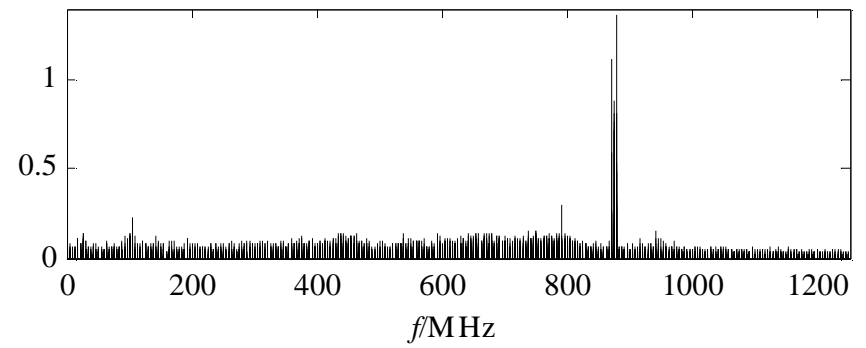

(a) Surface discharge

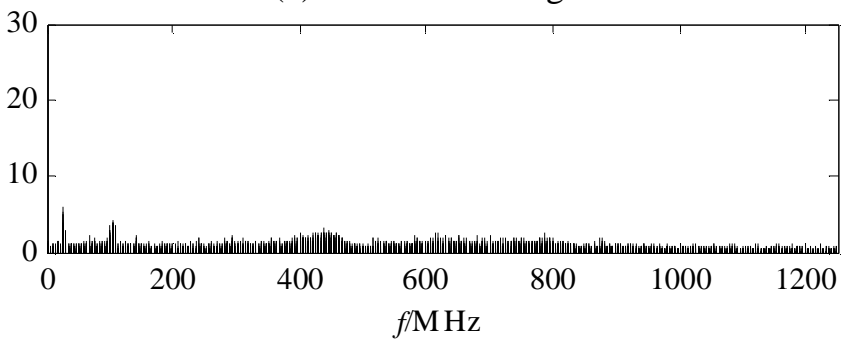

(b) Floating potential

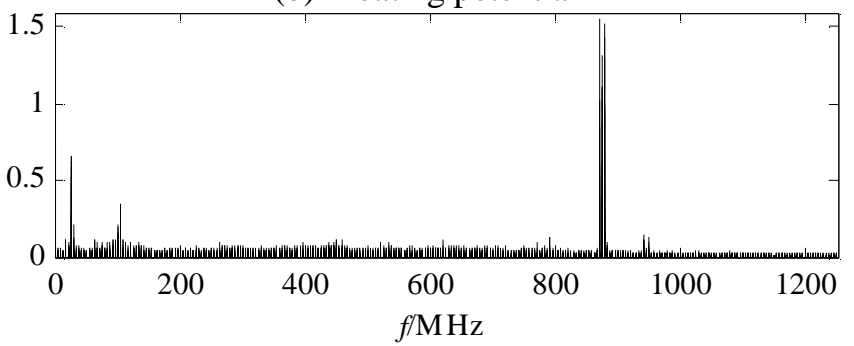

(c) Metal particles

Fig. 1 Frequency spectra of typical PD signals

As shown in Fig. 1, the frequency spectrum distribution of these three typical PD signals exhibits the following characteristics:

(1) The frequencies of various types of PD signals below $300 \mathrm{MHz}$ have similar components, with no values for identifying PD types.

(2) According to the practical experiences, the frequency spectra of the PD signals induced by metal particles and floating potential at the maximal amplitudes (at approximately $870 \mathrm{MHz}$ ) are actually the noise bands.

(3) The high frequency components at $900 \mathrm{MHz}$ or above impose little effect on the envelope of the signal's time domain waveform, and hence are also valueless.

Conclusively, a 12 order Butterworth band-pass filter with the pass band ranging from 300 to 850 $\mathrm{MHz}$ was used in the pre-filtering process with the purpose of achieving the UHF signals with high signal to noise (SNR) ratios.

\section{Acquisition of signal envelope}

Using the UHF method, the effective frequencies of the measured PD signals generally range from $300 \mathrm{MHz}$ to $1.5 \mathrm{GHz}$, i.e., they can be regarded as the high frequency oscillation signals. To gain an 
insight into the overall tendency of the high frequency signals in time domain, we should acquire the signal's envelope.

The common methods for extracting the signal envelope include Hilbert transform, demodulation filtering method, high pass absolute value demodulation and spline curve method. Demodulation filtering method extracts the envelope with respect to the middle line of the signal's positive half cycle, while the high pass absolute value method extracts the envelope regarding the signal's middle line. Thus, both methods cannot acquire the actual signal envelope. The principle of the interpolation point selection can hardly be determined for the spline curve method which has poor adaptability to various types of signals. Hence it was not adopted in the present work.

Finally, Hilbert transform was selected for acquiring the envelopes of UHF signals. Hilbert transform was first proposed by David Hilbert for solving the Riemann Hilbert problems of holomorphic function. It is a transform from one time domain to another, and now is quite mature and widely applied in the field of signal processing.

For a signal $x(t)$ with continuous time domain, the Hilbert transform is defined as the convolution of this signal with $h(t)=\frac{1}{\pi t}$, i.e.,

$$
\hat{x}(t)=H[x(t)]=x(t) * h(t)=\frac{1}{\pi} \int_{-\infty}^{+\infty} x(\tau) \cdot \frac{1}{t-\tau} \mathrm{d} \tau
$$

The analytic signal of the original signal $x(t)$ is defined as:

$$
a(t)=x(t)+j \hat{x}(t)
$$

The modulus of the analytic signal can be written as:

$$
E(t)=|a(t)|=\sqrt{x^{2}(t)+\hat{x}^{2}(t)}
$$

which is exactly the envelope of the original signal $x(t)$.

For a discrete signal sequence $x(n)$ with the length of $\mathrm{m}$ and the corresponding sequence $X(k)$ after fast Fourier transform (FFT), there is

$$
A(k)= \begin{cases}X(k), & k=0 \\ 2 X(k), & k=1,2,3, \ldots, \frac{m}{2}-1 \\ 0 & k=\frac{m}{2}, \frac{m}{2}+1, \ldots, m-1\end{cases}
$$

where $A(k)$ denotes the FFT sequence corresponding to the discrete analytic signal $a(n)$ in $x(n)$.

After the inverse fast Fourier transform (IFFT) on the acquired $A(k)$, the modulus was solved. Thus the envelope signal $E(n)$ can be obtained:

$$
E(n)=|a(n)|=\mid \operatorname{IFFT} A(k)] \mid
$$

\section{Extraction of envelope characteristic parameters}

After acquiring the envelopes of UHF signals through Hilbert transform, we can observe that the envelopes of different types of PD signals are similar to the double exponential function of an attenuated vibration in waveform, but have obvious differences in oscillation frequency and attenuation time constant. The types of PD signals can then be identified by extracting the data at several key points.

As shown in Fig. 2, we preliminarily extracted five characteristic values of the envelope of UHF PD signals. They are pulse peak $\mathrm{V}_{\text {top }}$, the first wave trough after the peak $\mathrm{V}_{\mathrm{v} 1}$, the first wave peak after the peak $\mathrm{V}_{\mathrm{p} 1}$, the second wave trough after the peak $\mathrm{V}_{\mathrm{v} 2}$ and the second wave peak after the peak $\mathrm{V}_{\mathrm{p} 2}$. 


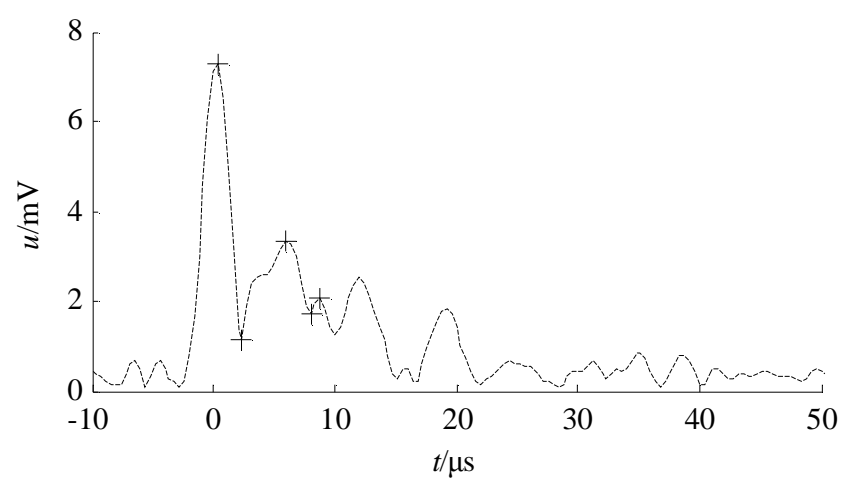

Fig. 2 Envelope of UHF PD signal and its characteristics

The differences in the distance between PD source and sensor and in PD type will affect the amplitude of UHF signals. Therefore, the above described original characteristic values should be first normalized, and then can be used as the characteristic parameters of PD signals.

\section{Construction of feature space}

With the computing speed and the algorithm's performance and intuitiveness taken into account, three characteristic parameters with the most favorable discrimination performance were selected to construct the feature space, i.e., to select three parameters with optimal discrimination performance in the set $\left\{\frac{V_{\mathrm{v} 1}}{V_{\text {top }}}, \frac{V_{\mathrm{p} 1}}{V_{\text {top }}}, \frac{V_{\mathrm{v} 2}}{V_{\text {top }}}, \frac{V_{\mathrm{p} 2}}{V_{\text {top }}}\right\}$ as the $\{x, y, z\}$ in feature space.

Then the concept of the differentiability of random variables was introduced.

Definition of the differentiability of two random variables, $a$ and $b$, at the same dimension:

$$
D_{a b}=\left|\frac{\bar{\lambda}_{a}-\bar{\lambda}_{b}}{\sigma_{a}+\sigma_{b}}\right|
$$

Suppose $\bar{\lambda}_{a}$ and $\bar{\lambda}_{\mathrm{b}}$ denote the average values of the samples of two variables, respectively; $\sigma_{a}$ and $\sigma_{b}$ denote the standard deviations of the unbiased samples. The larger the value of $\mathrm{D}_{\mathrm{ab}}$, the less likely the two characteristic parameters, $a$ and $b$, are confused.

It should be achieved as far as possible that the constructed 3D feature space is able to differentiate each type of PD signals, i.e., the emphasis should be laid on the global discrimination. Therefore, in the selection of characteristic parameters, we should abide by the following principle: the combination of the characteristic parameters with the poorest discrimination performance on the PDs of a certain or several types should be excluded.

Accordingly, the differentiability of each type of PD signals at each dimension of characteristic parameter was calculated in this article, so as to select the optimal combination to construct the feature space.

\section{Experiments and results}

\section{Experimental design}

To examine the effectiveness of the proposed method, an experimental platform was established for the UHF based PD detection for GIS, and various defect samples were designed and prepared for simulating three types of typical GIS insulation faults, namely, surface discharge, metal particles and floating potential. Additionally, the UHF signals of these three faults were detected in the experiments.

The experimental system consists of power supply, experimental model and PD detection device, as shown in Fig. 3. 


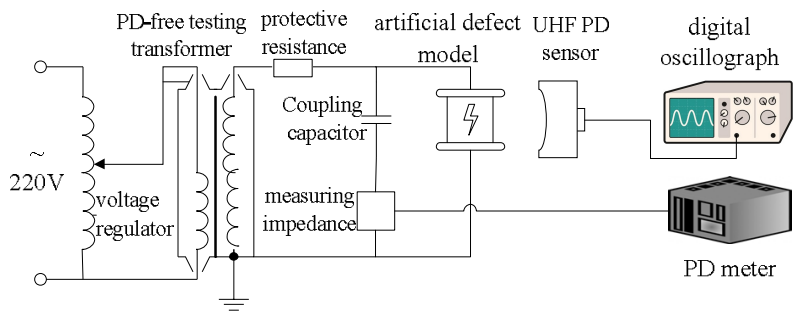

Fig. 3 Illustration of the experimental system

The power supply in the present experiments is composed of the control panel, voltage regulator, testing transformer and protective resistance. The testing transformer is a power frequency PD free testing transformer. A $5 \mathrm{k} \Omega$ protective resistance is used to restrict the output current during a flashover. The capacitance of the coupling capacitor is $1000 \mathrm{pF}$. The PD electromagnetic wave signal sensor uses the double arm Archimedean spiral antenna, with the bandwidth ranging from $300 \mathrm{MHz}$ to $1.5 \mathrm{GHz}$.

Using the established experimental device, the PD signal samples of three typical artificial defects surface discharge, floating potential and metal particles, were collected. Fig. 4 shows these UHF PD signals after preprocessing.

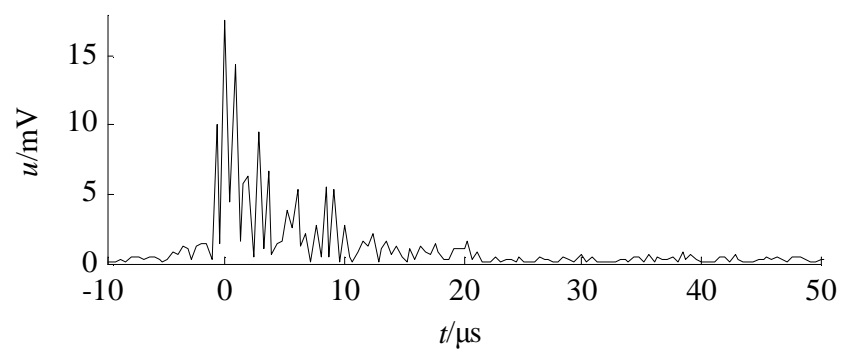

(a) Surface discharge

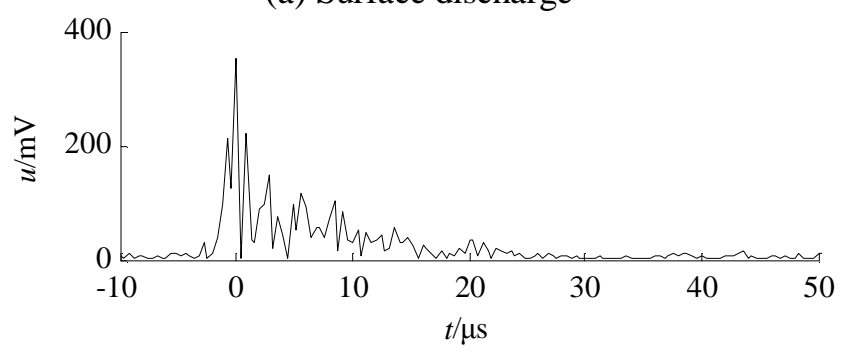

(b) Floating potential

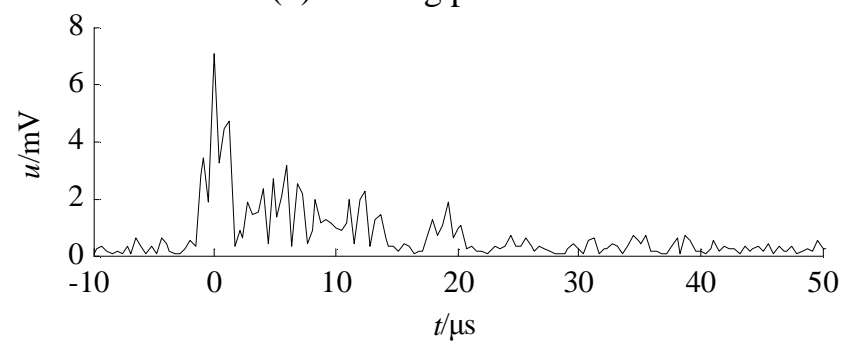

(c) Metal particles

Fig. 4 UHF PD signals after preprocessing

According to above described method, the signals were processed and the characteristic parameters of the waveform were extracted, with their distribution shown in Fig. 5. In the figure, type 1, type 2 and type 3 denote the PD signals of the artificial defects surface discharge, floating potential and metal particles, respectively. 


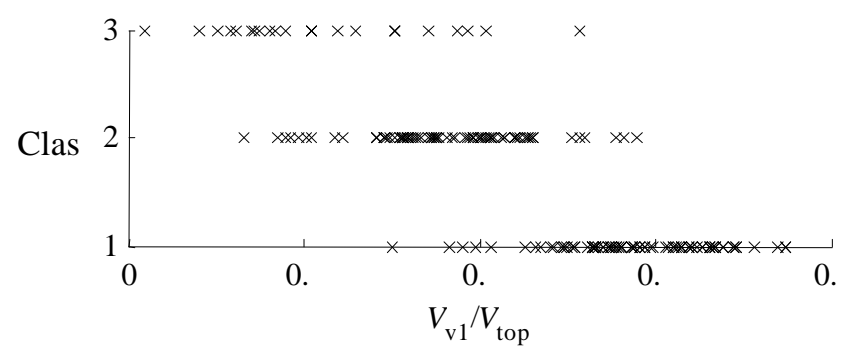

(a) Characteristic parameter 1

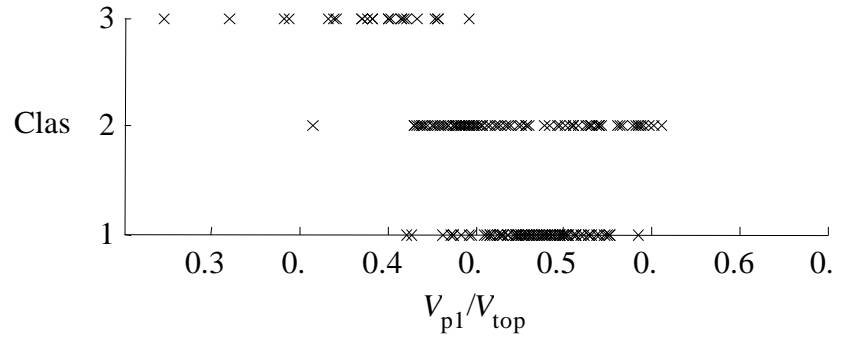

(b) Characteristic parameter 2

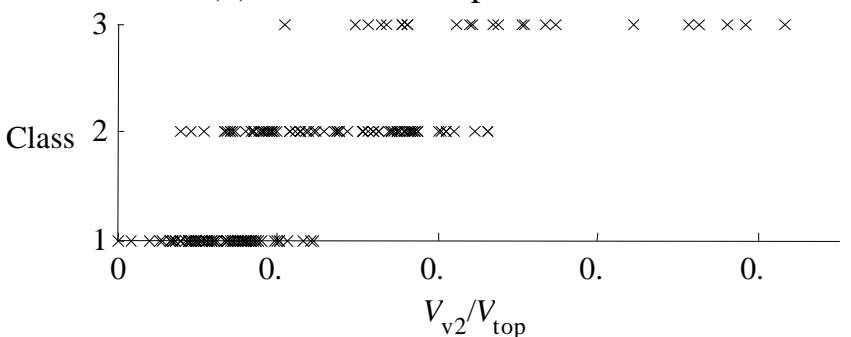

(c) Characteristic parameter 3

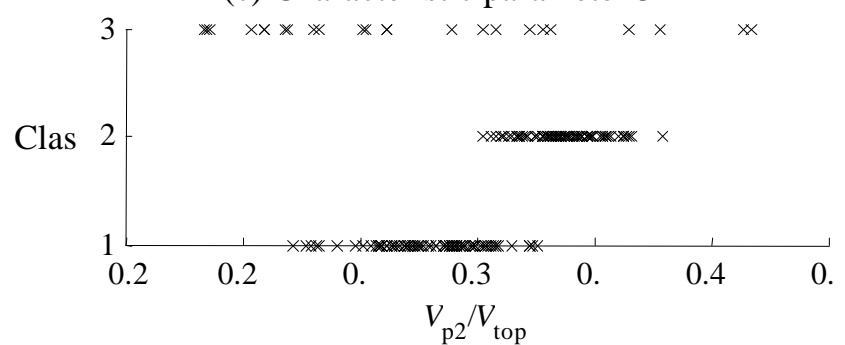

(d) Characteristic parameter 4

Fig. 5 Characteristic parameters of the envelope of UHF PD signal Table 1 lists the statistics of these four characteristic parameters.

Table 1 Characteristic parameters of three types of typical PDs

\begin{tabular}{|c|c|c|c|c|c|}
\hline \multicolumn{2}{|c|}{$\begin{array}{l}\text { Characteristic parameter } \\
\text { Term }\end{array}$} & $V_{\mathrm{v} 1} / V_{\text {top }}$ & $V_{\mathrm{pl}} / V_{\text {top }}$ & $V_{\mathrm{v} 2} / V_{\text {top }}$ & $V_{\mathrm{p} 2} / V_{\text {top }}$ \\
\hline \multirow{2}{*}{ 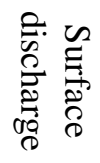 } & Average value & 0.2881 & 0.5332 & 0.0638 & 0.3303 \\
\hline & Standard deviation & 0.0432 & 0.0255 & 0.0237 & 0.0227 \\
\hline \multirow{2}{*}{ 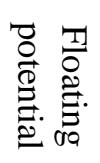 } & Average value & 0.1810 & 0.5214 & 0.1358 & 0.3874 \\
\hline & Standard deviation & 0.0440 & 0.0431 & 0.0477 & 0.0156 \\
\hline \multirow{2}{*}{ 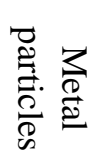 } & Average value & 0.1133 & 0.4377 & 0.2443 & 0.3237 \\
\hline & Standard deviation & 0.0597 & 0.0394 & 0.0864 & 0.0716 \\
\hline
\end{tabular}


Table 2 lists the differentiability of each characteristic parameter of the PD signals of different defects.

Table 2 Differentiability of each characteristic parameter

\begin{tabular}{|c|c|c|c|}
\hline $\begin{array}{c}\text { Characteristic } \\
\text { parameter }\end{array}$ & $\begin{array}{c}\text { Surface discharge } \\
\text { floating potential }\end{array}$ & $\begin{array}{c}\text { Floating potential } \\
\text { metal particles }\end{array}$ & $\begin{array}{c}\text { Metal particles } \\
\text { surface discharge }\end{array}$ \\
\hline$V_{\mathrm{v} 1} / V_{\text {top }}$ & 1.2285 & 0.6528 & 1.6987 \\
\hline$V_{\mathrm{p} 1} / V_{\text {top }}$ & 0.1716 & 1.0149 & 1.4725 \\
\hline$V_{\mathrm{v} 2} / V_{\text {top }}$ & 1.0082 & 0.8089 & 1.6389 \\
\hline$V_{\mathrm{p} 2} / V_{\text {top }}$ & 1.4932 & 0.7310 & 0.0697 \\
\hline
\end{tabular}

Table 3 lists the statistics of differentiability of different combinations of characteristic parameters. The data reflecting the poorest discrimination performance were highlighted with boldface.

Table 3 Differentiability of different combinations of characteristic parameter

\begin{tabular}{|c|c|c|c|}
\hline $\begin{array}{l}\text { EDifferentiability } \\
\text { Abandoned } \\
\text { characteristic } \\
\text { parameter }\end{array}$ & $\begin{array}{c}\text { Surface discharge } \\
\text { floating potential }\end{array}$ & $\begin{array}{c}\text { Floating potential } \\
\text { metal particles }\end{array}$ & $\begin{array}{c}\text { Metal particles } \\
\text { surface discharge }\end{array}$ \\
\hline$V_{\mathrm{v} 1} / V_{\text {top }}$ & 2.67 & $\mathbf{2 . 5 5}$ & 3.18 \\
\hline$V_{\mathrm{p} 1} / V_{\text {top }}$ & 3.73 & 2.89 & 2.43 \\
\hline$V_{\mathrm{v} 2} / V_{\text {top }}$ & 2.89 & 3.09 & $\mathbf{2 . 2 6}$ \\
\hline$V_{\mathrm{p} 2} / V_{\text {top }}$ & $\mathbf{2 . 4 1}$ & 3.17 & 3.83 \\
\hline
\end{tabular}

As shown in Table 3, the data reflecting the poorest discrimination performance did not fall into the column that abandons $\mathrm{V}_{\mathrm{p} 1} / \mathrm{V}_{\text {top }}$. Therefore, the characteristic parameters with favorable discrimination performance are $\mathrm{V}_{\mathrm{v} 1} / \mathrm{V}_{\text {top }}, \mathrm{V}_{\mathrm{v} 2} / \mathrm{V}_{\text {top }}$ and $\mathrm{V}_{\mathrm{p} 2} / \mathrm{V}_{\text {top }}$. Using the three characteristic parameters as the coordinates in a specific 3D space, as shown in Fig. 6, we can observe that the data are obviously classified into three types.

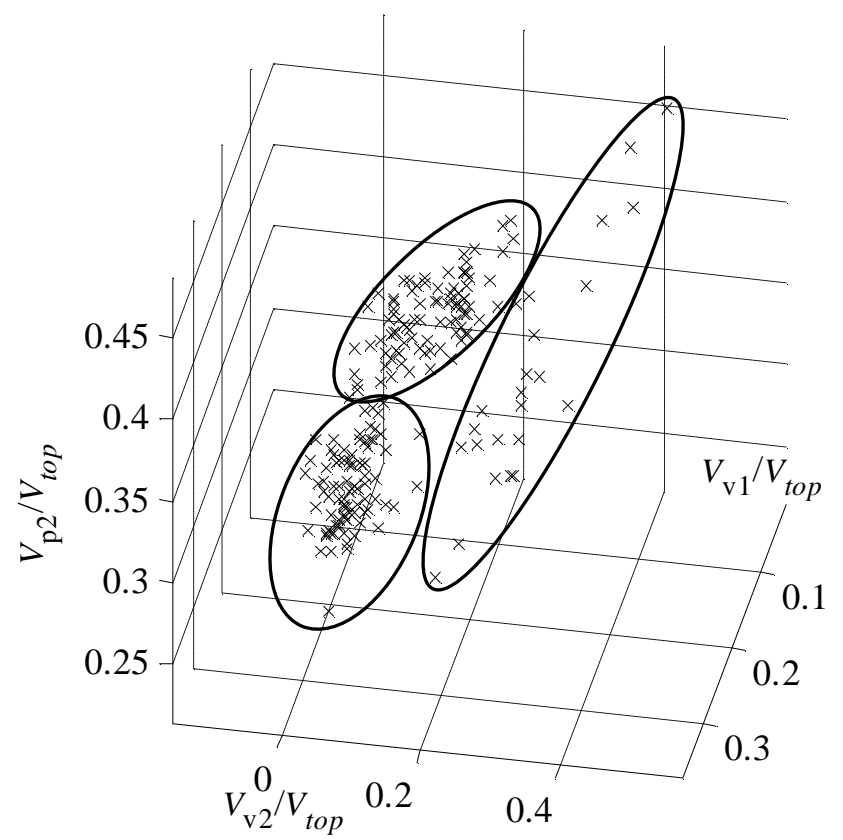

Fig. 6 Distribution of the data in 3D space

Using the above described characteristic parameters in combination with a simple three layer back propagation (BP) neural network, these three types of PD signals can be identified. The recognition results are shown in Table 4. 
Table 4 Confusion matrix based on the BP neutral network

\begin{tabular}{|c|c|c|c|c|}
\hline Output Input & $\begin{array}{c}\text { Surface } \\
\text { discharge }\end{array}$ & $\begin{array}{c}\text { Floating } \\
\text { potential }\end{array}$ & $\begin{array}{c}\text { Metal } \\
\text { particles }\end{array}$ & Accuracy \\
\hline $\begin{array}{c}\text { Surface } \\
\text { discharge }\end{array}$ & $42.6 \%$ & $0.1 \%$ & $0.0 \%$ & $98.8 \%$ \\
\hline $\begin{array}{c}\text { Floating } \\
\text { potential }\end{array}$ & $0.5 \%$ & $44.2 \%$ & $0.5 \%$ & $97.8 \%$ \\
\hline $\begin{array}{c}\text { Metal } \\
\text { particles }\end{array}$ & $0.0 \%$ & $0.0 \%$ & $11.7 \%$ & $100 \%$ \\
\hline $\begin{array}{c}\text { Recognition } \\
\text { ratio }\end{array}$ & $98.8 \%$ & $98.9 \%$ & $95.8 \%$ & $(98.5 \%)$ \\
\hline
\end{tabular}

As shown in Table 4, the top three columns in terms of recognition ratio with the BP neural network, which reflects the recognition capability of neural network on this type of signals, reflecting the reliability of the neural network output on this results. In the rest parts of the table, the total number of these three types of samples is $100 \%$. On the lower right corner of the table, the value represents the recognition effect of neural network for the whole samples. Here, the accuracy equals to the recognition ratio and also equals to the sum of the rest elements along the principal diagonal of the confusion matrix. Additionally, it equals to the percentage of the number of all accurate recognitions to the total number of samples. As can be observed, the pattern recognition performance is favorable, i.e., the selected characteristic parameters in this article are of great help to effective recognitions.

\section{Conclusions}

In this particle, based on the experiments on the PD signals of GIS defects, the algorithm for extracting the envelopes of UHF signals and the corresponding envelope characteristic parameters were investigated. In combination with a neural network, we analyzed the PD recognition technique for GIS based on the envelope characteristics of UHF signals, and the primary conclusions are listed below.

(1) The envelopes of the UHF PD signals of GIS acquired by Hilbert transform can effectively characterize different types of PDs.

(2) The extracted envelope characteristic parameters have obvious signal characterization abilities. In combination with the neural network, the extracted characteristic parameters can accurately identify several types of typical GIS insulation defects.

\section{References}

[1] DP Duan, GIS Partial Discharge Detection and Biomimetic Pattern Recognition Based on UHF Method, Shanghai: Shanghai Jiao Tong University, 2009: 1. .

[2] BF Hampton, RJ Meats. Diagnostic Measurements at UHF in Gas Insulated Substations [J]. IEE Proceedings C on GTD.: 1988, 135 (2): 137-145.

[3] Y Qian, CJ Huang, and XC Jiang et al., Present Situation and Prospect of Ultrahigh-frequency-Method-based Research of on-line Monitoring of Partial Discharge in Gas Insulated Switchgear, Power System Technology, 2005, 29 (1): 40.

[4] G Vaillancourt, R Malewski. Digital Acquisition and Processing of Partial Discharge during Acceptance Tests of HV Transformers [C]. Paper 88 WM049-9, IEEE/PES Winter Meeting 1988

[5] XY Zhang and SL Ma, Implementation of Digital Envelope, Journal of Changchun University of Science and Technology (Natural Science Edition), 2008, 31 (2): 118-120. 
[6] XS Zhang, YS Zhu, XX Cheng and Y M Cheng, A Method of Signal Envelope Extraction from Hilbert Transform to Wavelet Transform, Journal of Electronics, 1997, 19(1): 120-123.

[7] WR Si, JH Li, and P Yuan et al., The Fast Grouping Technique of PD Sequence Based on the Nonlinear Mapping of Pulse Shapes, Transactions of China Electrotechnical Society, 2009, 24(3): 216 221, 228.

[8] NC Sahoo, MMA Salama, Trends in Partial Discharge Pattern Classification: A Survey [J]. IEEE Transactions on Dielectrics and Electrical Insulation: 2005, 12 (2): 248-264.

[9] J Guo, GN Wu, XQ Zhang and W Shu, The Actuality and Perspective of Partial Discharge Detection Techniques, Transactions of China Electrotechnical Society, 2005, 20 (2).

[10] WJ Si, JH Li, and P Yuan et al., Detection and Recognition Technique for Multi-PD Source in GIS, Proceedings of the CSEE, 2009: 29 (16): 119 126.

[11] W Wang, CR Li, and YS Ding et al., Simulation on the Application of Detection Technique to Detecting UHF PD signals, High Voltage Technology, 2004, 30(2): 32 47. 